\title{
HYCANTHONE E OXAMNIQUINE NO TRATAMENTO DE CRIANÇAS PORTADORAS DE S. MANSONI*
}

\author{
Dra. Maria Zelia Rouquayrol; Y.M. Almoida; E.G. Oliveira; Z.F. Silva; V.A.M. Pinto \& J.E. Alencar * *
}

Os autores apresentam resultado de tratamento com Hycanthone e com Oxamniquine efetuado em grupos de 36 crianças de $6-15$ anos de idade, portadoras de S. mansoni, mais um grupo Controle (não portadores), de igual número perfazendo o total de 108 crianças.

Os grupos eram homogêneos quanto à idade, peso, altura, condições clínicas (forma intestinal moderada) e fatores sócio-econômico-sanitários. Eram todos residentes no perímetro irrigado da Curú-Recuperação, Pentecoste-Ceará, e filhos de funcionários do DNOCS.

Foi efetuado tratamento com Hycanthone $(2,3 \mathrm{~m} / \mathrm{kg}$ a $2,7 \mathrm{mg} / \mathrm{kg})$ mais placebo de Oxamniquine (Grupo A), com Oxamniquine $(11,4 \mathrm{mg} / \mathrm{kg}$ a $20 \mathrm{mg} / \mathrm{kg}$ ) mais placebo de Hycanthone (Grupo B) e placebo dos dois medicamentos para o grupo Controle (Grupo C). Foram realizados exames de sangue (hemograma, transaminases e fosfatases alcalinas), sumário de urina e coproscopias (Hoffman, Kato qualitativo e quantitativo). Após tratamento todos os casos ficaram sob observação durante 72 horas e mais seguimento clínico e laboratorial durante 4 meses. Efetuados teste de $t$ e de chi-quadrado todos os resu/tados são apresentados em tabelas e assim sumariados:

1) Hycanthone mostrou eficácia completa apresentando $100 \%$ como índice de cura.

2) Oxamniquine apresentou indice de cura de $80 \%$, sendo que os 7 pacientes não curados tiveram significativa redução do número de ovos viáveis; destes, após o 20 tratamento, persistiram apenas 3 eliminando ovos viáveis.

3) Efeitos colaterais do tratamento com Hycanthone foram os seguintes: dor local, febre, náuseas, vômitos, cefaléia, tontura e sonolência.

4) Oxamniquine quase não afetou as crianças do ponto de vista de efeitos colaterais demonstráveis, tendo apresentado alguns casos com febre moderada e transitória $e$ um caso com queixa de náusea.

5) Testes de função hepática revelaram alterações leves com aumento de TGO tanto no Grupo Hycanthone como no Grupo Oxamniquine (sem diferenca estatistica significativa, inclusive para o grupo Controle) e aumento discreto de TGP para ambos os grupos. Não houve aumento significativo das fosfatases para nenhuma das amostras.

6) Dada a dificuldade de empregar, para todas as crianças, a dosagem de $20 \mathrm{mg} / \mathrm{kg}$ por se tratar de cápsula não fracionável, sugere-se a forma farmacêutica em xarope na hipótese de que com o acréscimo das doses de Oxamniquine haja significativo aumento do Indice de cura. Que essa correção seja efetuada paralelamente a cuidadosos exames complementares a fim de se detectar a toxicidade a esse nfvel.

Trabalho realizado sob os auspício do DNOCS e do Centro de Ciências da Saúde da Universidade Federal do Ceará (Convênio PGE-15/75).

* Docentes do Departamento de Saúde Comunitéria e do Departamento de Patologia do Centro de Ciências da Saúde da Universidade Federal do Ceará.

Recebido para publicação em 18/2/1976. 


\section{INTRODUÇÃO}

Baseado em algumas considerações formuladas por Katz ${ }^{6}$, onde questões básicas são por ele levantadas (toxicidade das drogas, dados comparativos entre as drogas, estudos retrospectivos e prospectivos, etc.), foi elaborado o presente trabalho com os seguintes objetivos:

a) Fornecer dados comparativos sobre o valor terapêutico dos dois medicamentos atualmente empregados no combate à esquistossomose (Hycanthone e Oxamniquine).

b) Por em relevo as vantagens e as desvantagens no emprego dos referidos medicamentos em crianças que representam o grupo mais exposto ao risco de infecção esquistossomótica em áreas endêmicas.

\section{MATERIAL E MÉTODOS}

No presente trabalho todas as crianças, filhas de funcionários do DNOCS residentes no perimetro irrigado de Curu-Recuperação-Ceará, estavam situadas na faixa etária de 6 a 15 anos de idade subdivididas em três grupos de 36 , sendo do is de portadores de $S$. mansoni e o outro constituído de não portadores, perfazendo o total de 108 crianças.

Foram preenchidas fichas de família com itens sobre condições sócio-econômico-sanitárias contendo as seguintes informações: ! procedência da família, idade das crianças, seu peso e altura, qualidade da água, coleta de lixo, destino de dejetos, renda da família e tipo de habitação.

Obtendo-se anuência dos pais para iniciarmos o tratamento das crianças, todos os familiares foram informados da importância da participação ativa de cada membro na luta empreendida contra a esquistossomose, bem como foram fornecidas informações sobre o tratamento, demora e tipos de exames laboratoriais a que seriam submetidas as crianças para assim obtermos o máximo de cooperação e de rendimento no controle da endemia.

Antes da terapêutica contra $S$. mansoni iniciamos nosso trabalho examinando clinicamente com o máximo rigor todas as crianças que também foram tratadas com medicação polivalente contra as enteroparasitoses. Chamamos atenção para o fato de que todos os pacientes portavam a forma intestinal moderada.

Após homogeneização quanto à idade, altura, peso e condições clínicas, os grupos de portadores foram submetidos a sorteio aleatório e tratados, em ambulatório, com Hycanthone via intramuscular $(2,3$ a $2,7 \mathrm{mg} / \mathrm{kg})$ mais placedo de Oxamniquine (Grupo A), Oxamniquine via oral $(11,4$ a $20 \mathrm{mg} / \mathrm{kg})$ mais placebo de Hycanthone (Grupo B) e os não portadores foram tratados com placebo dos dois medicamentos (Grupo C).

A temperatura dos pacientes foi tomada antes do tratamento e após 6, 24 e 48 horas.

Após tratamento os 3 grupos ficaram sob observação durante 72 horas.

Foram efetuadas coproscopias (métodos de Hoffman, Kato qualitativo e quantitativo e exames seriados em meio de MIF) antes do tratamento e após um, dois e quatro meses respectivamente.

$\mathrm{Na}$ avaliação dos possíveis efeitos tóxicos dos medicamentos, utilizou-se a dosagem de transaminases (Karmen, $\mathrm{uK} / \mathrm{ml}$ ) e fosfatases alcalinas (Bessey, Lowry e Brock - 45 a $210 \mathrm{mU} / \mathrm{ml}$, faixa normal), bem como determinação de parâmetros hematológicos (hematimetria, hematócrito e hemoglobina e leucometria), tais valores sendo mensurados, para todas as crianças, antes, 72 hs e 4 meses após o início do tratamento.

Para a avaliação estatística de significância entre diferenças de médias amostrais, utilizou-se o teste " $t$ " de Student-Fisher. Considerando-se a grande variabilidade inter $e$ intragrupos dos valores de transaminasemia, o que reduziria a validade do teste " $\mathrm{t}$ ", comprovamos a homogeneidade das diversas amostras, para essa variável, lançando mão da prova não-paramétrica do $\chi^{2}$ (Chi-quadrado). Os níveis de significância adotados foram de $5 \%$.

\section{RESULTADOS E COMENTÁRIOS}

Variáveis básicas (idade, peso e estatura). A tabela 1 , descreve as características dessas variáveis nos grupos estudados. A tabela 2 analisa as variáveis, segundo as suas médias, desvios padrões, bem como os resultados do teste estatístico, que não mostrou diferença significativa para as diversas comparações. Considerou-se, dessa forma, a homogeneidade entre as amostras examinadas e, conseqüentemente, a validade do estabelecimento de comparações.

\section{Toxicidade}

Transaminases e fosfatases - 0 gráfico I e tabela 3 mostram a distribuição das transaminases e fosfatases nos 3 grupos, durante os diversos períodos (antes, $72 \mathrm{hs}$ e $4 \mathrm{~m}$ ), bem 
como a amplitude de variação de cada um desses valores. Verifica-se, em relação às fosfatases, que, em nenhum caso houve elevação dessa enzima (levar em conta os valores normais correspondentes a crianças). Já com respeito às transaminases, embora as médias correspondam a valores dentro do intervalo considerado normal, em algumas oportunidades o limiar de normalidade foi ultrapassado. A tabela 3 relaciona os casos onde, pelo menos em uma ocasião, houve aumento de TGO ou TGP; constata-se que são 19 crianças, das quais 3 pertencendo ao controle, 8 ao grupo Hycanthone e 8 ao grupo Oxamniquine.

Pode-se presumir que as alterações observadas no grupo controle sejam, pelo menos em parte, dependentes do estado de desnutrição crônica e infecções multiparasitárias a que estavam sujeitas essas crianças. Para os diversos períodos de observação, o teste de $\chi^{2}$ (chi-quadrado) não mostrou heterogeneidade entre os grupos. Silva e cols. ${ }^{12}$ tratando 48 crianças fazem referência ao aumento das transaminases sendo $14(35 \%)$ para TGO e $9(17,5 \%)$ para TGP. Cunha e Cançado ${ }^{4}$ relatam elevação das transaminases, especialmente TGO, logo após o tratamento com Hycanthonè.

Nosso estudo revelou discreto aumento das transaminases (TGO ou TGP), tanto em crianças tratadas com Hycanthone como em crianças tratadas com Oxamniquine, registrando-se apenas um único caso de elevação bastante acentuada de TGO em um paciente tratado com Oxamniquine (tabela 5).

\section{Valores hematologicos}

A tabela 4 resume os achados obtidos com relação aos parâmetros hematológicos fundamentais. Leucocitose moderada, com regressão observada aos 4 meses, foi vista em 11 pacientes, sendo 7 do grupo Hycanthome e 4 do grupo Oxamniquine. Mais freqüente, embora também em grau moderado, foi a tendência à leucopenia, principalmente no grupo Oxamniquine onde 11 crianças persistiram com cifras subnormais de leucócitos desde 72 hs até a última avaliação após o tratamento.

Com respeito à serie vermelha, não se destacou neñhuma alteração digna de registro.

Efeitos colaterais - Os efeitos colaterais mais comumente observados dentre as $\mathbf{3 6}$ crianças que tomaram Hycanthone foram dor local em 23 pacientes $(63,8 \%)$, náusea em 11 casos $(30,5 \%)$ e febre $(25 \%)$, assim distribuidos:

Apenas dor local (25\%), náusea, vômito e dor local $(13,7 \%)$, febre $(11,1 \%)$, náusea e dor local $(8,3 \%)$, febre e dor local $(5,6 \%)$, cefaléia e dor local $(5,6 \%)$, náusea, tontura, vômito $e$ febre $(5,6 \%)$, cefaléia, febre e dor local $(5,6 \%)$, sonolência $(5,6 \%)$, náusea e febre $(2,8 \%)$. Vários autores ${ }^{1,7,8}$ fazem referência à dor local e náusea como efeitos colaterais mais comuns. Além disto, em nosso estudo, foram registrados estados febrís de $37^{\circ} \mathrm{C}$ a $38^{\circ} \mathrm{C}$ em 5 casos e febre acima de $38^{\circ} \mathrm{C}$ em 4 pacientes.

Quanto às 36 crianças medicadas com Oxamniquine, apenas uma queixou-se de náusea

Efeitos colaterais (isolados ou associados) dentre 36 crianças tratadas com Hycanthone

\begin{tabular}{l|c|c}
\hline \multicolumn{1}{c}{ EFEITOS COLATERAIS } & No & $\%$ \\
\hline Dor local & 9 & 25,0 \\
Náusea, vômito e dor local & 5 & 13,7 \\
Febre & 4 & 11,1 \\
Náusea e dor local & 3 & 8,3 \\
Febre e dor local & 2 & 5,6 \\
Cefaléia e dor local & 2 & 5,6 \\
Náusea, tontura, vômito e febre & 2 & 5,6 \\
Cefaléia, tontura, febre e dor local & 2 & 5,6 \\
Sonolência & 2 & 5,6 \\
Náusea e febre & 1 & 2,8 \\
Nenhuma queixa & 4 & 11,1 \\
\hline TOTAL & 36 & 100,0 \\
\hline
\end{tabular}




\section{TABELA 1}

Variáveis básicas (Idade, peso, altura) determinadas entre 108 crianças submetidas a tratamento

\begin{tabular}{|c|c|c|c|}
\hline \multirow{2}{*}{$\begin{array}{l}\text { Grupos Etários } \\
\text { (Anos de Idade) }\end{array}$} & \multicolumn{3}{|c|}{ Número de Crianças } \\
\hline & Hycanthone & Oxamniquine & Controle \\
\hline $\begin{array}{r}6-7 \\
8-9 \\
10-11 \\
12-13 \\
14-15\end{array}$ & $\begin{array}{r}3 \\
5 \\
7 \\
11 \\
10 \\
36\end{array}$ & $\begin{array}{r}7 \\
2 \\
8 \\
9 \\
10 \\
36\end{array}$ & $\begin{array}{r}4 \\
10 \\
10 \\
8 \\
4 \\
36\end{array}$ \\
\hline \multirow{2}{*}{$\begin{array}{l}\text { Peso } \\
\text { (Kg) }\end{array}$} & \multicolumn{3}{|c|}{ Número de Crianças } \\
\hline & Hycanthone & Oxamniquine & Controle \\
\hline $\begin{array}{l}15-20 \\
21-26 \\
27-32 \\
33-38 \\
39-44 \\
45-50 \\
51-56 \\
\text { Total }\end{array}$ & $\begin{array}{r}7 \\
11 \\
6 \\
5 \\
3 \\
4 \\
- \\
36\end{array}$ & $\begin{array}{c}8 \\
7 \\
8 \\
6 \\
3 \\
4 \\
- \\
36\end{array}$ & $\begin{array}{r}7 \\
6 \\
11 \\
7 \\
3 \\
1 \\
1 \\
36\end{array}$ \\
\hline \multirow{2}{*}{$\begin{array}{l}\text { Altura } \\
(\mathrm{m})\end{array}$} & \multicolumn{3}{|c|}{ Número de Crianças } \\
\hline & Hycanthone & Oxamniquine & Controle \\
\hline $\begin{array}{l}1,00-1,10 \\
1,11-1,21 \\
1,22-1,32 \\
1,33-1,43 \\
1,44-1,54 \\
1,55-1,65\end{array}$ & $\begin{array}{l}4 \\
5 \\
8 \\
9 \\
5 \\
5\end{array}$ & $\begin{array}{r}3 \\
8 \\
5 \\
10 \\
5 \\
5\end{array}$ & $\begin{array}{r}1 \\
10 \\
7 \\
10 \\
8 \\
0\end{array}$ \\
\hline Total & 36 & 36 & 36 \\
\hline
\end{tabular}




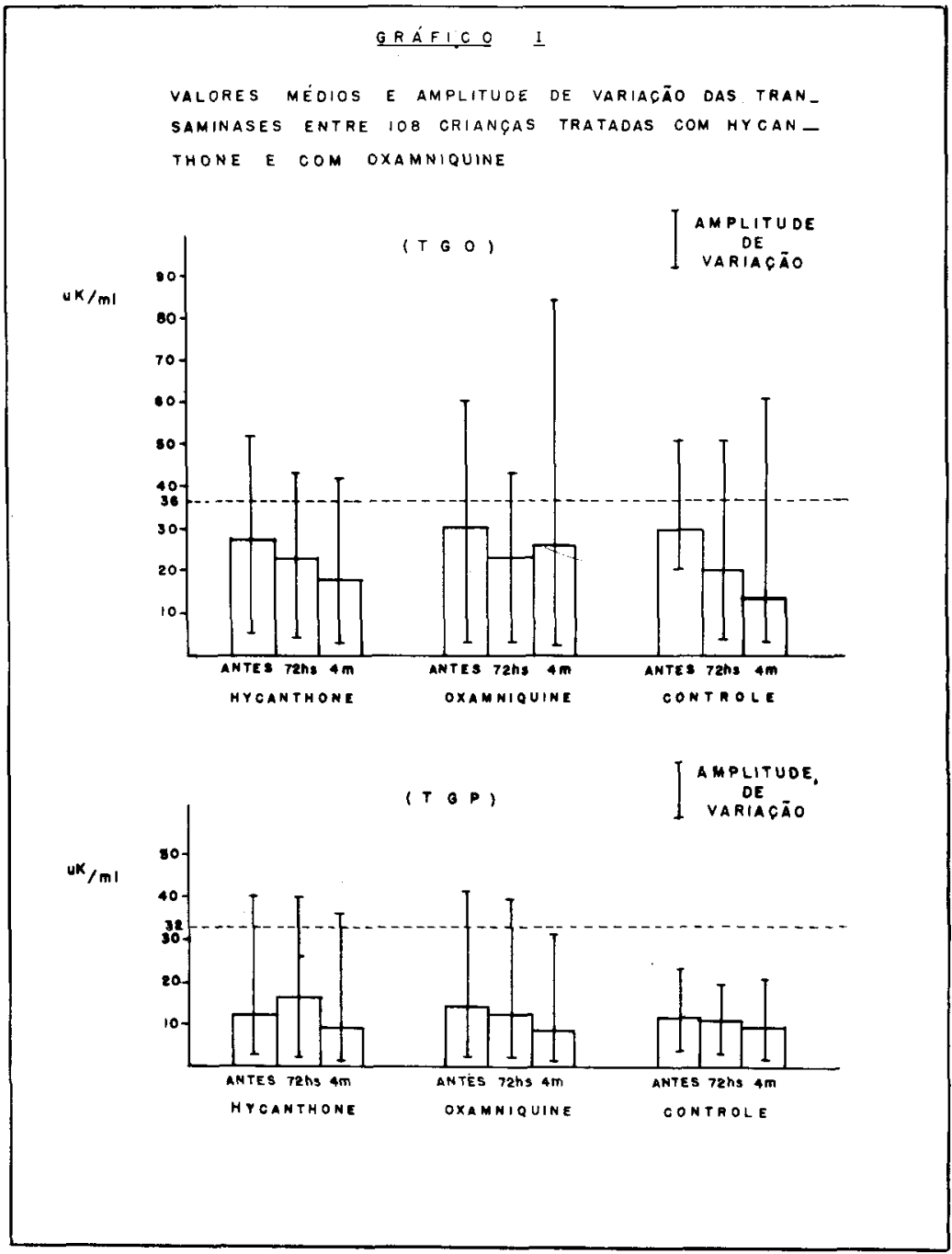

Tabela 2

Valores Médios das variáveis básicas (Idade, peso e altura) determinadas entre 108 crianças tratadas por Hycanthone e Oxamniquine

\begin{tabular}{l|c|c|c}
\hline \multirow{2}{*}{ Grupos * } & \multicolumn{3}{|c}{ Variáveis Básicas } \\
\cline { 2 - 4 } & $\begin{array}{c}\text { Idade }^{* *} \\
\text { (Anos) }\end{array}$ & $\begin{array}{c}\text { Peso } \\
(\mathrm{kg})\end{array}$ & $\begin{array}{c}\text { Altura * } \\
(\mathrm{m})\end{array}$ \\
\hline Hycanthone & $11,6 \pm 2,5$ & $29,3 \pm 9,1$ & $1,33 \pm 0,17$ \\
Oxamniquine & $11,2 \pm 2,9$ & $29,6 \pm 9,8$ & $1,33 \pm 0,17$ \\
Controle & $10,3 \pm 2,3$ & $29,5 \pm 8,8$ & $1,32 \pm 0,14$ \\
\hline
\end{tabular}

* Diferenças entre médias das variáveis nos três grupos sem significância estatística $(p<0,05)$.

* Média \pm desvio padrão. 
TABELA 3

Testes de Função Hepática em 72 crianças tratadas com Hycanthone e Oxamniquine e 36 do grupo controle

\begin{tabular}{|c|c|c|c|c|c|c|c|c|c|}
\hline \multirow{2}{*}{ Grupos } & \multicolumn{3}{|c|}{$\mathrm{TGO} u \mathrm{~K} / \mathrm{ml}$} & \multicolumn{3}{|c|}{$\mathrm{TGP} \mathrm{uK} / \mathrm{ml}$} & \multicolumn{3}{|c|}{ Fosfatases Alcalinas $\mathrm{mU} / \mathrm{ml}$} \\
\hline & Antes & $72 \mathrm{hs}$ & 4 meses & Antes & 72 hs & 4 meses & Antes & 72 hs & 4 meses \\
\hline Hycanthone & $27,5 \pm 11,5^{(*)}$ & $23,5 \pm 11,9$ & $18,7 \pm 9,0$ & $12,7 \pm 7,6^{(*)}$ & $16,6 \pm 8,7$ & $9,3 \pm 6,8$ & $106,8 \pm 36,4^{(*)}$ & $81,4 \pm 28,8$ & $89,5 \pm 23,0$ \\
\hline Oxamniquine & $30,0 \pm 10,1$ & $23,3 \pm 1.1,1$ & $257 \pm 12,8$ & $14,2 \pm 8,2$ & $11,8 \pm 9,7$ & $8,4 \pm 6,3$ & $99,3 \pm 30,4$ & $94,3 \pm 38,1$ & $83,6 \pm 26,3$ \\
\hline Controle & $30,6 \pm \quad 7,9$ & $19,0 \pm 8,0$ & $12,9 \pm 13,9$ & $10,5 \pm 4,8$ & $10,5 \pm 7,9$ & $8,6 \pm 4,7$ & $110,7 \pm 28,0$ & $94,2 \pm 29,5$ & $74,5 \pm 22,4$ \\
\hline
\end{tabular}

(*) Média \pm desvio Padrão.

\section{TABELA 4}

Valores Hematológicos

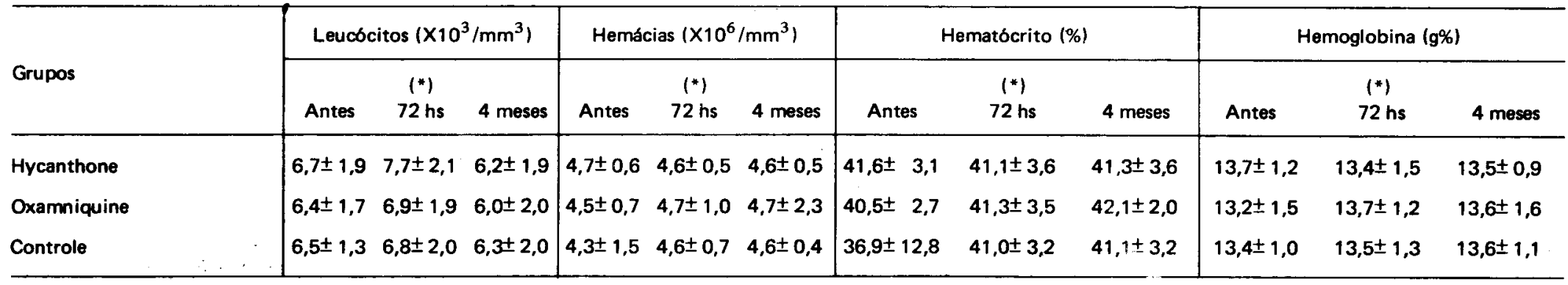

(*) Média \pm Desvio Pạdrão. 
TABELA 5

Relação de 19 casos com alterações das transaminases dentre 108 crianças examinadas.

\begin{tabular}{|c|c|c|c|c|c|c|c|}
\hline \multirow{2}{*}{ Pacientes } & \multirow{2}{*}{ Grupo } & \multicolumn{3}{|c|}{ TGO (uK/ml) } & \multicolumn{3}{|c|}{$\mathrm{TGP}(\mathrm{uK} / \mathrm{ml})$} \\
\hline & & Antes & $\begin{array}{l}72 \\
\text { hs }\end{array}$ & $4 \mathrm{~m}$ & Antes & $\begin{array}{l}72 \\
\text { hs }\end{array}$ & $4 m$ \\
\hline 1 - E.S. B. & Oxamniquine & 61 & 51 & 78 & 8 & 9 & 13 \\
\hline $2-\mathrm{E} . \mathrm{C}$. & Oxamniquine & 28 & 40 & 13 & 11 & 6 & 3 \\
\hline 3 - L. C. R. & Hycanthone & 22 & 43 & 6 & 3 & 19 & 1 \\
\hline$A-J$. L. S. & Oxamniquine & 25 & 43 & 10 & 8 & 25 & 1 \\
\hline 5 - J. L. S. & Hycanthone & 25 & 20 & 12 & 4 & 35 & 6 \\
\hline $6-$ T. C. F. & Hycanthone & 5 & 41 & 20 & 6 & 16 & 2 \\
\hline 7 - J. T. P. & Oxamniquine & 28 & 24 & 46 & 18 & 16 & 22 \\
\hline $8-$ M. T. P. & Hycanthone & 43 & 43 & 29 & 25 & 25 & 18 \\
\hline $9-$ M. V.P. & Hycanthone & 47 & 43 & 12 & 8 & 32 & 6 \\
\hline $10-$ A.S. N. & Hycanthone & 51 & 39 & 42 & 18 & 18 & 22 \\
\hline 11 - B.S. N. & Oxamniquine & 47 & 39 & 42 & 18 & 18 & 30 \\
\hline $12-$ M. C. S. N. & Controle & 44 & 21 & 46 & 16 & 11 & 18 \\
\hline $13-$ M. F. S. N. & Controle & 39 & 28 & 37 & 13 & 8 & 15 \\
\hline $4-1.0$ & Oxamniquine & 43 & 24 & 37 & 16 & 11 & 12 \\
\hline 15 - L. $O$. & Hycanthone & 39 & 32 & 32 & 13 & 16 & 36 \\
\hline $16-$ J. I. O. & Oxamniquine & 31 & 39 & 20 & 22 & 18 & 18 \\
\hline $17-$ J.E. F.R. & Controlè & 20 & 51 & 61 & 16 & 16 & 9 \\
\hline 18 - F. C.S. F. & Oxamniquine & 25 & 10 & 85 & 8 & 9 & 9 \\
\hline 19 - М. С. O. & Hycanthone & 31 & 32 & 42 & 16 & 14 & 12 \\
\hline
\end{tabular}

TABELA 6

Distribuição de ovos viáveis (por grama de fezes) de $S$. mansoni entre dois grupos de crianças tratadas por Hycanthone e por Oxamniquine

\begin{tabular}{c|cc|cc}
\hline $\begin{array}{l}\text { Ovos Viáveis } \\
\text { (P. grama) }\end{array}$ & $\begin{array}{c}\text { Grupo A } \\
\text { Antes }^{*}\end{array}$ & $\begin{array}{c}\text { (Hycanthone) } \\
\text { Após }\end{array}$ & $\begin{array}{c}\text { Grupo B (Oxamniquine) } \\
\text { Antes }\end{array}$ & $\begin{array}{c}{ }^{*} \\
\text { Após }\end{array}$ \\
\hline $20 \vdash 200$ & 31 & 0 & 29 & 6 \\
$200 \vdash 380$ & 2 & 0 & 6 & 1 \\
$380 \vdash 500$ & 0 & 0 & 0 & 0 \\
$500 \vdash 740$ & 1 & 0 & 0 & 0 \\
$740 \vdash 920$ & 1 & 0 & 0 & 0 \\
$920 \vdash 1100$ & 0 & 0 & 1 & 0 \\
$1100 \vdash 1280$ & 0 & 0 & 0 & 0 \\
$1280 \vdash 1460$ & 0 & 0 & 0 & 0 \\
$1460 \vdash 1640$ & 0 & 0 & 0 & 0 \\
$1640 \vdash 1820$ & 1 & 0 & 0 & 0 \\
\hline Total & 36 & 0 & 36 & 7 \\
\hline
\end{tabular}

* A distribuição antes do tratamento não apresenta diferença estatística significante entre os grupos (teste t, $\mathbf{p}<0,05)$. 
TABELA 7

Resultados do tratamento com Oxamniquine em segundo esquema após 4 meses da primeira dose

\begin{tabular}{|c|c|c|c|c|c|c|c|c|c|c|c|c|c|}
\hline \multirow[b]{2}{*}{ Paciente } & \multirow{2}{*}{$\begin{array}{l}\text { Idade } \\
\text { (Anos) }\end{array}$} & \multirow[t]{2}{*}{ Sexo } & \multirow{2}{*}{$\begin{array}{c}\text { (kg) } \\
\text { Peso } \\
\text { à } \\
\text { 1ạ dose }\end{array}$} & \multirow{2}{*}{$\begin{array}{c}\text { (kg) } \\
\text { Peso } \\
\text { à } \\
\text { 2a dose }\end{array}$} & \multicolumn{2}{|c|}{$\begin{array}{c}\text { Doses aplicadas } \\
\text { (mg/kg) }\end{array}$} & \multicolumn{2}{|c|}{$\begin{array}{l}\text { Efeitos } \\
\text { Colaterais }\end{array}$} & \multicolumn{3}{|c|}{ Número de ovos viáveis/grama } & \multicolumn{2}{|c|}{$\begin{array}{l}\text { Redução de ovos } \\
\text { viáveis (\%) }\end{array}$} \\
\hline & & & & & 19 & $2 \underset{a}{a}$ & $\begin{array}{c}19 \\
\text { Dose }\end{array}$ & $\begin{array}{c}2^{a} \\
\text { Dose }\end{array}$ & $\begin{array}{c}\text { Antes do } \\
\text { Trata- } \\
\text { mento }\end{array}$ & $\begin{array}{c}4 \text { meses } \\
\text { aposs } \\
1 \text { a dose }\end{array}$ & $\begin{array}{c}3 \text { meses } \\
\text { apos } \\
29 \text { dose }\end{array}$ & $\begin{array}{c}\text { Apos 1a } \\
\text { Dose. }\end{array}$ & $\begin{array}{c}\text { Após 2a } \\
\text { Dose }\end{array}$ \\
\hline M.A.R.P. & 7 & $\mathbf{F}$ & 18 & 20 & 13,9 & 12,5 & Nenh. & Nenh. & 322 & 23 & 0 & 92,7 & 100,0 \\
\hline S.H.J.S. & 11 & $\mathbf{F}$ & 22 & 28 & 11,4 & 17,8 & Nenh. & Nenh. & 299 & 276 & 23 & 7,7 & 91,7 \\
\hline M.T.P. & 15 & $M$ & 53 & 54 & 14,2 & 13,9 & Nenh. & Nenh. & 207 & 23 & 0 & 88,9 & 100,0 \\
\hline J.I.O. & 15 & $\mathbf{M}$ & 45 & 51 & 16,7 & 14,7 & Nenh. & Nenh. & 115 & 23 & 23 & 80,0 & 0,0 \\
\hline N.C. & 11 & $\mathbf{F}$ & 21 & 25 & 11,9 & 20,0 & Nenh. & Nenh. & 72 & 23 & 0 & 68,1 & 100,0 \\
\hline F.L.J.S. & 12 & $F$ & 27 & 31 & 18,5 & 16,1 & Nenh. & Nenh. & 23 & 23 & 23 & 0,0 & 0,0 \\
\hline M.C.L.S. & 10 & $\mathbf{F}$ & 20 & 25 & 12,5 & 20,0 & Nenh. & Nenh. & 23 & 23 & 20 & 0.0 & 100,0 \\
\hline
\end{tabular}

TABELA 8

Valores do TGO e TGP do primeiro e do segundo tratamento com Oxamniquine

\begin{tabular}{|c|c|c|c|c|c|c|c|c|c|c|c|c|c|c|c|}
\hline \multirow{3}{*}{ Paciente } & \multicolumn{5}{|c|}{ Transaminases (TGO) } & \multicolumn{5}{|c|}{ Transaminases (TGP) } & \multicolumn{5}{|c|}{ Fosfatases Alcalinas } \\
\hline & \multicolumn{3}{|c|}{ Primeiro Tratamento } & \multicolumn{2}{|c|}{$\begin{array}{l}\text { Segundo } \\
\text { Tratamento }\end{array}$} & \multicolumn{3}{|c|}{ Primeiro Tratamento } & \multicolumn{2}{|c|}{$\begin{array}{l}\text { Segundo } \\
\text { Tratamento }\end{array}$} & \multicolumn{3}{|c|}{ Primeiro Tratamento } & \multicolumn{2}{|c|}{$\begin{array}{c}\text { Segundo } \\
\text { Tratamento }\end{array}$} \\
\hline & Antes & Apos $72 \mathrm{hs}$ & Após 4m & Antes & Após 4m & Antes & Após 72hs & Após 4m & Antes & Após 4m & Antes & Apos 72hs & Após 4m & Antes & Apos 4m \\
\hline M.A.R.P. & 25 & 32 & 32 & 33 & 17 & 20 & 16 & 13 & 18 & 3 & 102 & 80 & 56 & 34 & 56 \\
\hline S.H.J.S. & 32 & 35 & 17 & 20 & 1 & 16 & 28 & 17 & 15 & 6 & 56 & 58 & 56 & 14 & 42 \\
\hline M.T.P. & 47 & 28 & 24 & 24 & 3 & 22 & 16 & 41 & 22 & 3 & 100 & 92 & 66 & 24 & 42 \\
\hline J.I.O. & 8 & 13 & 17 & 20 & 10 & 29 & 16 & 6 & 15 & 12 & 48 & 50 & 46 & 52 & 52 \\
\hline N.C. & 22 & 21 & 20 & 37 & 3 & 9 & 11 & 12 & 12 & 3 & 80 & 60 & 60 & 36 & 54 \\
\hline F.L.J.S. & 22 & 21 & 28 & 24 & 13 & 25 & 25 & 3 & 18 & 15 & 116 & 62 & 40 & 20 & 50 \\
\hline M.C.L.S. & 44 & 21 & 27 & 29 & 25 & 16 & 11 & 6 & 15 & 6 & 66 & 40 & 92 & 18 & 50 \\
\hline
\end{tabular}


è 5 registraram febre de 37 a $37,5^{\circ} \mathrm{C}$ e apenas 3 (8\%) com febre de 38 a $38,5^{\circ} \mathrm{C}$. Vários autores $3,5,10$ também fazem referência a este tipo de reação em maior ou menor intensidade.

No Grupo Controle foram registrados 1 caso de dor local e outro de cefaléia, tóntura e vômito. Cinco crianças (14\%) tiveram febre de 37 a $37,5^{\circ} \mathrm{C}$ e nenhuma acima de $37,5^{\circ} \mathrm{C}$.

Eficácia - Dentre 36 crianças tratadas com Hycanthone, na dosagem de $2,3 \mathrm{mg} / \mathrm{kg}$ a $2,7 \mathrm{mg} / \mathrm{kg}$, todas apresentaram exame de fezes seriados (MIF) negativos para $S$. mansoni em coproscopias realizadas no 10 , 2 9 e 4 ب̣ mês após o tratamento. Todas as 36 crianças do grupo Controle apresentaram resultados negativos quanto à presença de $S$. mansoni à 1ạ, 2ạ, e 4ạ coproscopias realizadas.

Dentre 36 crianças tratadas com Oxamniquine (cápsulas), na dosagem de $11,4 \mathrm{mg} / \mathrm{kg}$ a $20 \mathrm{mg} / \mathrm{kg}$, sete $(20 \%)$ continuaram a eliminar ovos viáveis em todas as coproscopias seriadas (MIF) realizadas no 19 , 29 e 49 mês de seguimento.

Na impossibilidade de fracionarmos a cápsula para dosagens mais consentâneas com o nosso desiderato de mantê-las entre 15 a $20 \mathrm{mg} / \mathrm{kg}$, e não dispondo da forma em xarope, tivemos que efetuar a dosagem (em uma ou duas tomadas) levando em conta o peso da criança e o número de cápsulas a ingerir, tendo-se o cuidado de não ultrapassar a recomendação oficial de $20 \mathrm{mg} / \mathrm{kg}$.

Na tabela 6 estão relacionados o número de ovos viáveis por grama de fezes e as freqüências relativas para cada classe antes e após o tratamento.

Evidencia-se que a maioria das crianças ( 31 do grupo Hycanthone e 29 para Oxamniquine) estava eliminando menos do que 200 ovos por grama de fezes antes do tratamento. Nas classes de mais de $\mathbf{2 0 0}$ ovos vamos notar freqüência de 5 crianças para o grupo A (Hycanthone) e 7 para o grupo $B$ (Oxamniquine) havendo, inclusive, um paciente no grupo $A$ que eliminava entre 1640 a 1820 ovos viáveis por grama de fezes. Teste $t$ efetuado entre os grupos não apresentou diferença estat ística significante quanto à eliminação de ovos antes do tratamento.

$O$ controle da cura efetuado durante 4 meses demonstrou que já a partir do 19 mês os exames se negativaram completamente no grupo tratado com Hycanthone. No grupo tratado com Oxamniquine, 7 crianças continuaram a eliminar ovos viáveis em todas as coproscopias seriadas realizadas no 19,29 e 49 mês após o tratamento específico.
Após 4 meses novo esquema foi elaborado para as $\mathbf{8}$ crianças não curadas, pesando-se outra vez os pacientes e ministrando-Ihes o medicamento em uma ou duas tomadas de acordo com o número de cápsulas.

Os resultados apresentados na tabela 7 indicam claramente que houve redução apreciável do número de ovos viáveis após o 1 e e $\circ 2$ ? tratamento. Chama-se atenção para o fato de que a paciente S.H.J.S., que tomou a menor fração $(11,4 \mathrm{mg} / \mathrm{kg})$, teve redução de ovos viáveis em apenas $7,7 \%$ após o 19 tratamento, e que tendo-se-Ihe aumentado a dose para 17,8, no 20 tratamento (permitindo o aumento por causa do novo peso da paciente), a redução neste novo esquema foi de $91,7 \%$.

Neste caso a orientação que nos ocorre para a melhoria da eficiência de Oxamniquine seria o de aumentar a dosagem e utilizar outra forma farmacêutica (xarope, por exemplo).

A outra paciente F.L.J.S., apesar de ter ingerido alta dose no 19 tratamento $(18,5 \mathrm{mg} / \mathrm{kg})$ não apresentou redução nesta nem tão pouco no 2 Q esquema, permanecendo com índice de redução igual a 0,0\%.

Uma explicação para este fato seria a da possível ocorrência de cepas resistentes ao medicamento, ou então por se tratar de infecção mais recente.

$\mathrm{Na}$ tabela 8 se registram os valores de transaminases e fosfatases nesse grupo de pacientes; nota-se que em apenas um caso houve elevação discreta de transaminase (TGP).

Em suma: Hycanthone revelou 100\% de eficácia pois nenhuma das 36 crianças tratadas continuou eliminando ovos viáveis após o tratamento (controle de cura ao $19,2 \%$ e 4 Q mês). Já a Oxamniquine revelou eficácia em $80 \%$ dentre 36 crianças tratadas em que 7 continuaram eliminando ovos viáveis após o tratamento. Em um segundo esquema de tratamento o índice de cura elevou-se a $92 \%$. Para o primeiro tratamento os nossos resultados são semelhantes ao de Rees e cols ${ }^{11}$ que referem $77 \%$ de cura entre 53 escolares por eles estudados. Resultados totalmente diversos encontraram Clarke e cols ${ }^{2} \mathrm{em}$ cujo trabalho pouquissimas curas radicais foram obtidas, a não ser redução apreciável na eliminação de ovos viáveis. 


\section{AGRADECIMENTOS}

Agradecemos a prestimosa colaboração do Dr. Danísio Dalton da Rocha Corrêa - Ex-Diretor do Centro de Ciências da Saúde e do Dr. Anibal Santos - Diretor da SUCAM, que nos colocaram transporte à disposição bem como pelas sugestões por eles apresentadas.

Ao Dr. José Hirton Dantas Carneiro Assistente da D.A.P. - DNOCS, nossos sinceros agradecimentos pelo apoio concedido às nossas reivindicações relacionadas a pessoal auxiliar, sem o qual não teria sido possível a execução do presente estudo.

Ao Dr. Roberto Alves Gurgel - Gerente do Perímetro Irrigado de Curu-Recuperação nossos efusivos agradecimentos pela colaboração irrestrita em todas as fases do trabalho.

Nossa gratidão às famílias de Pentecoste pela solidariedade e propósito de nos ajudarem a atingir os objetivos na luta contra a esquistossomose.

\section{SUMMARY}

The authors present their experience in the treatment of children infected with S. mansoni using Hycanthore (2.3 to $2.7 \mathrm{mg} / \mathrm{kg}$ body weight) and Oxamniquine capsules $(11.4$ to $20.0 \mathrm{mg} / \mathrm{kg}$ body weight); a control group was given placebo.

Hycanthone cured 100\% of the 36 children given the drug; Oxamniquine capsules cured $80 \%$ of the 36 children treated and a cure rate of $92 \%$ was achieved with a second oxamniquine treatment course. Side-effects were most frequently observed with Hycanthone: no significant liver test function a/terations were detected with both drugs.

The authors suggest the use of oxamniquine syrup for the treatment of children in order to obtain better results with more adequate dosages according to the body weight.

\section{REFERÊNCIAS BIBLIOGRÁFICAS}

1. BINA, J.C. \& PRATA, A - Hycanthone no tratamento da esquistossomose em uma área rural com baixo índice de transmissão da doença. Gaz. Med. Bahia 70; 127-130, 1970.

2. CLARKE, V.V.; BLAIR, D.M. \& WEBER, M. - Ensaios de Oxamniquine intramuscular nas infecções por $S$. haematobium e $S$. mansoni na Rodésia. Rev. Inst. Med. Trop. São Paulo, 15: 159-164, 1973.

3. COURA, J.R.; ARGENTO, C.A.; FIGUEIREDO, W \& QUEIROZ, G.C. - Experiência com a Oxamniquine - UK 4271 - no tratamento da esquistossomose mansoni. Rev. Inst. Med. Trop., São Paulo 15: 126-131, 1973.

4. CUNHA, A.S. \& CANÇADO, J.R. - Tratamento Clínico. In: CUNHA, A.S. da Esquistossomose mansoni. São Paulo, Editora Univ. S. Paulo, 1970. Cap. 9, p. 327-382.

5. COUTINHO, A.; DOMINGUES, A.L.C. \& BONFIM, J.R.A. - Tratamento da esquistos- somose mansonica com Oxamniquine, Rev. Inst. Med. Trop., São Paulo, 15: 104-119, 1973.

6. KATZ, N. - Novos esquistossomicidas: recentes progressos e necessidades de pesquisa. 16p. mimeografadas. Trabalho apresentado ao XI Congresso da Sociedade de Medicina Tropical, Rio de Janeiro, 1975.

7. NOLETO, P.A.; NEVES, P.F.; MAGALHÃES, O. \& SILVA, J.M. - Esquistossomose: 1000 pacientes tratados. A Folha Médica, 70, supl. esp., 15-18, $197,5$.

8. OLIVEIRA, P.A. - Controle da Esquistossomose em áreas selecionadas do Rio Grande do Norte. A Folha Médica, 70, supl. especial, 1-13, 1975.

9. ORGANIZAÇÃO MUNDIAL DE SAÚDE Comite de Expertos - La lucha contra la esquistosomiasis. Ginebra, OMS, 1973. 52p (Serie de Informes Tecnicos no 515).

10. PRATA, A; FIGUEIREDO, J.F.M.; BRANDT, P.C. \& LAURIA, L. - Oxamni- 
quine em dose única intramuscular no tratamento da esquistossomose mansoni. Rev. Inst. Med. Trop. São Paulo, 15: 132-142, 1973.

11. REES, P. H.; ROBERTS, J.M.D.; WOODGER, B.A. \& PAMBA, H.O. - Oxamniquine intramuscular no tratamento da esquistosso- mose mansoni em Quênia. Rev. Inst. Med. Trop. São Paulo, 15: 169, 1973.

12. SILVA, L.C. da; SETTE JR, H.; CHAMONE, D.A.F.; ALQUEZAR, A.S. \& MONTEIRO, A.A. - Oxamniquine no tratamento da esquistossome mansônica em área não endêmica. Rev. Inst. Med. T.rop. São Paulo, 15: 143-147, 1973. 